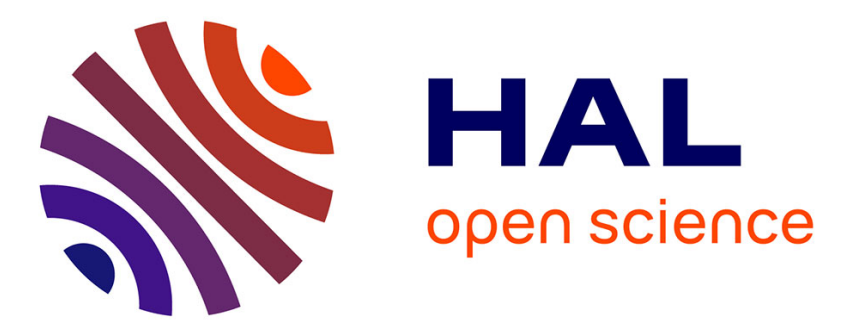

\title{
Deciphering ascending thoracic aortic aneurysm hemodynamics in relation to biomechanical properties
}

Giuseppe de Nisco, Paola Tasso, Karol Calò, Valentina Mazzi, Diego Gallo, Francesca Condemi, Solmaz Farzaneh, Stéphane Avril, Umberto Morbiducci

\section{- To cite this version:}

Giuseppe de Nisco, Paola Tasso, Karol Calò, Valentina Mazzi, Diego Gallo, et al.. Deciphering ascending thoracic aortic aneurysm hemodynamics in relation to biomechanical properties. Medical Engineering \& Physics, 2020. hal-03139655

\section{HAL Id: hal-03139655 https://hal.science/hal-03139655}

Submitted on 12 Feb 2021

HAL is a multi-disciplinary open access archive for the deposit and dissemination of scientific research documents, whether they are published or not. The documents may come from teaching and research institutions in France or abroad, or from public or private research centers.
L'archive ouverte pluridisciplinaire HAL, est destinée au dépôt et à la diffusion de documents scientifiques de niveau recherche, publiés ou non, émanant des établissements d'enseignement et de recherche français ou étrangers, des laboratoires publics ou privés. 
Title page

\section{Deciphering ascending thoracic aortic aneurysm}

\section{hemodynamics in relation to biomechanical properties}

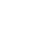

Giuseppe De Nisco ${ }^{1}$, Paola Tasso $^{1}$, Karol Calò $^{1}$, Valentina Mazzi

${ }^{1}$ Polito ${ }^{B I O}$ Med Lab, Department of Mechanical and Aerospace Engineering, Politecnico di Torino, Turin, Italy

2 Mines Saint-Etienne, Université de Lyon, INSERM, U 1059 SAINBIOSE, F - 42023 Saint-Etienne France

(1)

2

13

${ }^{\dagger}$ Address for correspondence:

Umberto Morbiducci, Ph.D.

Department of Mechanical and Aerospace Engineering, Politecnico di Torino

Corso Duca degli Abruzzi, 24 - 10129 Turin, Italy

Tel.: +390110906882

Fax: +390115646999

E-mail: umberto.morbiducci@polito.it 


\section{Abstract}

The degeneration of the arterial wall at the basis the ascending thoracic aortic aneurysm (ATAA) is a complex multifactorial process, which may lead to clinical complications and, ultimately, death. Individual genetic, biological or hemodynamic factors are inadequate to explain the heterogeneity of ATAA development/progression mechanisms, thus stimulating the analysis of their complex interplay.

Here the disruption of the hemodynamic environment in the ATAA is investigated integrating patient-specific computational hemodynamics, CT-based in vivo estimation of local aortic stiffness and advanced fluid mechanics methods of analysis. The final aims are (1) deciphering the ATAA spatiotemporal hemodynamic complexity and its link to near-wall topological features, and (2) identifying the existing links between arterial wall degeneration and hemodynamic insult. Technically, two methodologies are applied to computational hemodynamics data, the wall shear stress (WSS) topological skeleton analysis, and the Complex Networks theory. The same analysis was extended to the healthy aorta.

As main findings of the study, we report that: (1) different spatiotemporal heterogeneity characterizes the ATAA and healthy hemodynamics, that markedly reflect on their WSS topological skeleton features; (2) a clear link (stronger than canonical WSS-based descriptors) emerges between the variation of contraction/expansion action exerted by WSS on the endothelium along the cardiac cycle, and ATAA wall stiffness. The findings of the study suggest the use of advanced methods for a deeper understanding of the hemodynamics disruption in ATAA, and candidate WSS topological skeleton features as promising indicators of local wall degeneration. 


\section{Highlights}

53

54 ascending aorta hemodynamics, reflecting on wall shear stress topological skeleton.

55

56 aortic aneurysms stiffness.

57

58

59

60

61

62

63

64

- Different levels of spatiotemporal heterogeneity characterize the aneurysmal and healthy

- Peculiar wall shear stress topological skeleton features are linked to local ascending thoracic

- The topological shear variation index, a measure of wall shear stress luminal

8 contraction/expansion action variation along the cardiac cycle, is an indicator of local aortic

wall degradation, performing better than canonical wall shear stress-based descriptors of flow

disturbances.

- Wall shear stress topological skeleton analysis, combined with Complex Networks theory,

2 contributes to better determine whether arterial wall degeneration, in combination with

3 hemodynamic insult, leads to aneurysmal progression/rupture. 


\section{Introduction}

Ascending thoracic aortic aneurysm (ATAA) is an unphysiological localized ballooning of the aorta at the ascending segment proximal to the brachiocephalic trunk, which can grow in size and eventually rupture, leading to serious complications and death. The common clinical treatment of ATAA consists in surgical repair by replacing the diseased aortic segment with a synthetic graft $[1,2]$. Due to significant mortality rates associated to prophylactic surgery (3-5 \%) [1], indications for surgical treatment depend on the estimated maximum aortic diameter, with a suggested critical threshold value of $5.5 \mathrm{~cm}$ (except for patients with Marfan syndrome) [2-4]. However, the diameter-based criterion for surgical intervention has been widely recognized as inadequate $[5,6]$ To find complementary or alternative markers of ATAA risk of rupture, a plethora of studies have focused on, e.g., genetic, biological, structural and biomechanical factors involved in the ATAA wall dilatation/degeneration [7-13]. However, current evidence is still insufficient to rely on biomechanical factors alone $[10,12]$, and rather suggests a possible relation between ATAA hemodynamics and wall mechanical properties, which might better explain the complexity of ATAA development and progression.

In particular, the analysis of aortic hemodynamics, with the assessment of distinguishable intravascular flow features and wall shear stress (WSS) distribution on the luminal surface as obtained from in vivo [14-17] and patient-specific computational studies [18-22], have been suggested to derive markers of rupture risk, giving the supposed link between hemodynamic features and ATAA wall mechanical properties. For instance, previous follow-up studies reported a significant association between the luminal exposition to relatively low WSS values and ascending aorta wall weakening and dilatation in ATAA patients $[21,23,24]$.

In this context, we propose a framework combining patient-specific computational fluid dynamics (CFD), advanced fluid mechanics analysis and personalized in vivo estimates of the local aortic 
89

90

91

92

93

94

95

96

97

98

99

100

101

102

103

104

105

106

107

stiffness. The aims are to interpret the complex role played by local hemodynamics in ATAA and to decipher the existing links between near-wall features, intravascular flow structures and ATAA wall stiffness. More specifically, changes in hemodynamics associated with pathologic aortic dilatation were analyzed by applying two recently proposed methodologies on two subjectspecific ( 1 healthy - 1 diseased) CFD models. The first approach relies on the analysis of WSS vector field topological skeleton, essentially consisting of a collection of critical points (i.e., locations at the luminal surface of the vessel where the WSS vanishes), and special lines which separate the luminal surface into areas of different WSS behavior, ultimately identifying where an expansion/contraction action is exerted by WSS on the endothelium (which is expected to have biological relevance) [25][26]. The second one exploits Complex Networks (CNs) theory to describe the spatiotemporal heterogeneity in near-wall and intravascular flow patterns, revealing information embedded in hemodynamic quantities waveforms (along the cardiac cycle) and useful to describe nonlinear phenomena involving them [27]. Both approaches were combined here to relate near-wall hemodynamics and intravascular flow patterns, which are expected to markedly affect WSS features $[28,29]$. Finally, ATAA local stiffness was obtained through a recently proposed inverse method based on gated CT scans [30]. Spatial correlations with WSS features were extracted from the topological skeleton analysis to ultimately associate ATAA complex hemodynamic features and wall mechanical properties. 
109 One 36 years old healthy subject with no history of cardiovascular diseases, and one 59 years old 110 subject with bicuspid aortic valve (BAV), moderate aortic valve insufficiency and a $6.03 \mathrm{~cm} \times 5.95$ $111 \mathrm{~cm}$ ATAA were enrolled in the study after informed consent. An overview of the methods applied 112 in this study is provided in Figure 1 and detailed onwards.

\section{Medical imaging and geometry reconstruction}

114 The two subjects were imaged by a 3T MR scanner (Siemens Magnetom Prisma) without contrast 115 agent, using the same 4D flow MRI protocol exhaustively detailed elsewhere [22]. Based on 4D 116 flow MRI images, the 3D geometries of each subject were reconstructed using CRIMSON

117 (CardiovasculaR Integrated Modelling and SimulatiON) [31]. The resulting geometries included the ascending thoracic aorta, the aortic arch, the descending thoracic aorta and the supra-aortic vessels (brachiocephalic artery - BCA, left common carotid artery - LCCA, and left subclavian artery - LSA, as shown in Figure 1). Additionally, the diseased subject underwent ECG gated CT imaging in order to identify ATAA wall local stiffness with a recently proposed inverse method [30] detailed below.

A recently proposed non-invasive inverse approach was applied here to identify aortic wall stiffness for the diseased subject. Briefly, ATAA geometries at ten phases along the cardiac cycle, including diastolic and systolic phases, were (1) reconstructed from gated CT scans using MIMICS

127 (v.10.01, Materialise NV), and (2) meshed ensuring a set of nodes attached to the same material points at all the recorded cardiac phases. Based on the Fourier series analysis of temporal changes in position of each node, the local strain distribution was reconstructed and the wall stiffness at 
every location was derived by satisfying the local equilibrium equation under the acting tensions.

131 The strategy applied to estimate local wall stiffness in vivo is exhaustively detailed elsewhere [30].

132 Computational hemodynamics

133 The finite element-based open-source code SimVascular (http://simvascular.github.io/) was

134 adopted to solve the governing equations of fluid motion in the two models. In detail, the Navier-

135 Stokes equations, in their discretized form and under unsteady flow conditions, were solved using

136 a stabilized finite element method supporting the use of linear tetrahedral elements (P1-P1) in the

137 SimVascular flow solver for velocity and pressure [32]. Newtonian blood rheology (with prescribed

138 constant density and dynamic viscosity respectively equal to $1050 \mathrm{~kg} \mathrm{~m}^{-3}$ and $0.0035 \mathrm{~Pa} \mathrm{~s}$ ) and rigid

139 wall with no-slip condition were assumed. To ensure a grid-independent solution, based on a

140 mesh sensitivity analysis, an average tetrahedral element size of $1.05 \cdot 10^{-3} \mathrm{~m}$ with a near-wall

141 refinement consisting of 12 tetrahedral boundary layers with a decreasing ratio of 0.90 was

142 adopted. The resulting computational grids consisted of 4.13 and 7.97 million elements for healthy

143 and diseased model, respectively.

144 Boundary conditions were prescribed using patient-specific 4D flow MRI measurements. In detail,

145 measured fully 3D phase velocity data were extracted along the cardiac cycle and imposed in

146 terms of Dirichlet inflow boundary conditions (BCs) at the ascending aorta (AAo) inlet section (as

147 detailed in [33]). As regards outflow BCs, 4D flow MRI measured flow rates were prescribed at the

148 supra-aortic vessels [34] in terms of fully developed velocity profiles. A three element Windkessel

149 model was prescribed as BC at the descending aorta (DAo) outlet section, by applying a 3D-OD

150 coupling scheme [22,31]. The three element Windkessel model components (i.e., the impedance -

$151 Z_{C}$, the distal resistance $-R$, and the capacitor $-C$ ) were tuned as described in [22], obtaining

152 patient specific values for the healthy $\left(Z_{C}=1.36 \cdot 10^{7} \mathrm{~kg} \mathrm{~m}^{-4} \mathrm{~s}^{-1} ; R=2.28 \cdot 10^{8} \mathrm{~kg} \mathrm{~m}^{-4} \mathrm{~s}^{-1} ; C=1.50 \cdot 10^{-8}\right.$ 
$\left.153 \mathrm{~kg}^{-1} \mathrm{~m}^{4} \mathrm{~s}^{2}\right)$ and the pathologic subject $\left(Z_{C}=5.32 \cdot 10^{6} \mathrm{~kg} \mathrm{~m}^{-4} \mathrm{~s}^{-1} ; R=9.02 \cdot 10^{7} \mathrm{~kg} \mathrm{~m}^{-4} \mathrm{~s}^{-1} ; C=3.18 \cdot 10^{-8}\right.$

$\left.154 \mathrm{~kg}^{-1} \mathrm{~m}^{4} \mathrm{~s}^{2}\right)$

155 On the simulated flow fields in the healthy and diseased models, advanced post-processing tools were applied to better decipher the aortic hemodynamics complexity.

\section{Deciphering aortic hemodynamics - WSS topological skeleton analysis}

Based upon dynamical systems theory, the topological skeleton of a vector field is made of fixed points and manifolds, where: (1) a fixed point is a point where the vector field vanishes; (2) vector field manifolds, connecting fixed points, identify contraction/expansion regions (respectively, stable/unstable manifolds).

Here, a very recently proposed Eulerian method was considered to analyze the topological skeleton of the WSS vector field across the aortic luminal surface [25]. Based on the Volume Contraction Theory, it has been demonstrated [25] that specific features of the WSS vector field topological skeleton, i.e., WSS manifolds, can be easily captured using the divergence of the normalized WSS vector:

$$
\mathrm{DIV}=\nabla \cdot \boldsymbol{\tau}_{u}=\nabla \cdot\left(\frac{\boldsymbol{\tau}}{|\boldsymbol{\tau}|}\right)
$$

where $\boldsymbol{\tau}_{u}$ is the unit vector of WSS vector $\boldsymbol{\tau}$. Eq. (1), neglecting the WSS vector magnitude variation, identifies the WSS spatial contraction/expansion configuration patterns [25]: negative values of DIV identify contraction regions, approximating attracting manifolds; positive values of DIV identify expansion regions, approximating repelling manifolds (Figure 2a).

According to the scheme proposed elsewhere for the complete WSS topological skeleton extraction [25], the identification of WSS fixed points location at the luminal surface was performed by computing a mesh-independent topological index, named Poincaré index. Then, the identified fixed points were classified using the eigenvalues of the Jacobian matrix, which provide information about fixed points nature (Figure 2). Technically, the adopted eigenvalue-based 
criterion allowed to distinguish between fixed point node or focus configurations. The combinations of the Poincaré index values and of eigenvalues $\lambda_{i}(i=1,2,3)$ of the Jacobian matrix corresponding to the possible WSS fixed points configurations are summarized in Figure $2 \mathrm{~b}$. As a first step, here the WSS topological skeleton of the cycle-average WSS vector field $\overline{\mathbf{\tau}}$ at the luminal surface of both the healthy and diseased model was analyzed. The unsteady nature of the WSS vector field fixed points along the cardiac cycle was investigated using the WSS fixed points weighted residence time along the cardiac cycle [25]:

$$
R T \nabla_{x_{f p}}(e)=\frac{\bar{A}}{A_{e}} \frac{1}{T} \int_{0}^{T} \mathbb{I}_{e}\left(\boldsymbol{x}_{f p}, t\right)\left|(\nabla \cdot \boldsymbol{\tau})_{e}\right| d t
$$

where $x_{f p}(t)$ is the WSS fixed point position at time $t \in[0, T], e$ is the generic triangular element of the superficial mesh of area $A_{e}, \bar{A}$ the average surface area of all triangular elements of the superficial mesh, II is the indicator function and $(\nabla \cdot \tau)_{e}$ is the instantaneous WSS divergence.

Eq. (2) quantifies the fraction of cardiac cycle spent by a fixed point inside a generic mesh surface element $e$ on the aortic luminal surface emphasizing the contribution of fixed points surrounded by a marked local contraction/expansion action. In fact, from a physical point of view, the term $\left|(\nabla \cdot \tau)_{e}\right|$ in eq. (2) allows to measure the strength of the contraction/expansion action of shear on the wall.

Additionally, here a quantity based on WSS divergence, named Topological Shear Variation Index (TSVI), is introduced to measure the amount of variation in WSS contraction and expansion action exerted at the luminal surface of the vessel. To do that, the root mean square of the normalized WSS divergence is computed as follows:

$$
\operatorname{TSVI}=\left\{\frac{1}{T} \int_{0}^{T}\left[\nabla \cdot\left(\boldsymbol{\tau}_{u}\right)-\overline{\nabla \cdot\left(\boldsymbol{\tau}_{u}\right)}\right]^{2} d t\right\}^{1 / 2}
$$

Roughly speaking, eq. (3) allows to localize regions on the luminal surface of the vessel exposed to large variations in the shear contraction/expansion action exerted by the flowing blood along the 
200

201

202

203

cardiac cycle.

Deciphering aortic hemodynamics - Complex Networks analysis

The spatiotemporal heterogeneity of intravascular and near-wall flow patterns was here investigated applying a recently introduced approach based on the application of the CNs theory to computational hemodynamics data $[27,35]$, where the nodes of the network are represented by the waveforms, along the cardiac cycle, of specific hemodynamic quantities at the nodes of the aortic computational grid.

Here, the CNs-based analysis was applied to the WSS vector magnitude $(|\tau|)$ waveforms along the cardiac cycle, whereas intravascular hemodynamics was described in terms of axial velocity ( $V_{a x}$ ) and kinetic helicity density $\left(H_{k}\right)$. In detail, $V_{a x}$ is the component of blood velocity vector along the local axial direction, identified as the direction of the tangent to the vessel's centerline [36]. By definition, $V_{a x}$ is representative of the main flow direction. $H_{k}$ is defined as the internal product between local velocity and vorticity vectors and its sign is an indicator of the (right- or left-handed) direction of rotation of helical blood flow patterns. The latter descriptor was involved in the analysis because of the established physiological significance of helical blood flow in main arteries $[29,37,46,38-45]$.

For both investigated aortic models, three different CNs were built, starting from $V_{a x}, H_{k}$ and $|\tau|$ time-histories, respectively. In the here adopted approach [27], two generic nodes $i$ and $j$ of the $\mathrm{CN}$ are connected by a topological link $\{i, j\}$ if the Pearson correlation coefficient $\mathrm{R}_{i j}$ between the waveforms of each considered hemodynamic quantity at the two nodes is larger than a given threshold $\widehat{\mathrm{R}}$. In this sense, each $\mathrm{CN}$ can be represented by its adjacency matrix $\mathrm{A}_{i j}$ containing the information about node connectivity as follows: 
Here, for each aortic model, and for each of the three $\mathrm{CNs}$ based on the hemodynamic quantities $V_{a x}, H_{k}$ and $|\tau|$, respectively, the median values of the single $\mathrm{R}_{i j}$ distributions were set as threshold values $\widehat{R}$ to build up adjacency matrices, as proposed elsewhere $[27,35]$.

Three metrics, based on $A_{i j}$, were used to characterize the structure of each $\mathrm{CN}$. In detail, here we considered the degree centrality $D C_{i}$ of node $i$, defined as the percentage of nodes of the network connected to node $i$, i.e., the so-called neighbors of $i$ :

$$
D C_{i}=100\left(\frac{1}{N-1} \sum_{j=1}^{N} A_{i j}\right)=100\left(\frac{1}{N-1} d c_{i}\right)
$$

In eq. (5) $d c_{i}$ is the non-normalized degree centrality of node $i$. To allow comparisons between the healthy and the diseased model networks, characterized by different number of nodes $(N), d c_{i}$ was normalized to the total number $(N-1)$ of possible neighbors of $i$. In summary, for a specific hemodynamic descriptor, $D C_{i}$ measures the fraction of time-histories correlated above threshold with the time-history in node $i$, quantifying the spatiotemporal homogeneity/heterogeneity of the considered hemodynamic quantity waveform in each node, with respect to the whole network (i.e., the fluid volume for $V_{a x}$ and $H_{k}$, or the luminal surface for $|\tau|$ ).

The second CNs metric used in this study was applied to the $V_{a x^{-}}$and $H_{k}$-based networks to provide a quantitative measure of the anatomical length of persistence of the correlation of axial velocity and helical flow patterns inside the aorta. To do that, the so-called normalized average Euclidean distance $\left(A E D_{i}\right)[27,47]$ of each node $i$ of the network from all its neighbors $n(i)$ was evaluated, defined as:

$$
A E D_{i}=\frac{1}{D_{\max }} \frac{\sum_{j \in n(i)} l_{i j}}{d c_{i}},
$$

where $D_{\max }$ is the maximum AAo diameter in the region upstream of the proximal supra-aortic trunk, and $l_{i j}$ is the Euclidean distance between neighbor nodes $i$ and $j$. In eq. (6), $A E D_{i}$ was 
normalized with respect to $D_{\max }$, in order to account for AAo geometric variability between the healthy and the diseased model. To investigate the effect that the presence of the ATAA has on the anatomical extent of the spatiotemporal correlations between $V_{a x}$ and $H_{k}$ structures, in both models $A E D_{i}$ was computed only in the aortic region from the inlet section to the first supra-aortic vessel bifurcation point.

The length of persistence of the correlation of WSS spatiotemporal patterns at the aortic luminal surface was evaluated in topological terms in the $|\tau|$-based network of the two aortic models. This was done computing the average shortest path length $\left(\overline{S P L}_{i}\right)$ of node $i$, defined as:

$\overline{S P L}_{i}=\frac{1}{N-1} \sum_{j \in V, i \neq j} d_{i j}$

where $V$ is the set of nodes of the network, and $d_{i j} \in Z$ is the shortest topological distance between nodes $i$ and $j$, i.e., the minimum number of links connecting node $i$ with node $j$ [48] (Figure 1). In synthesis, $\overline{S P L}_{i}$ is a measure of the topological length of the correlation persistence of the $|\tau|$ waveform at each luminal surface node (i.e., the smaller the $\overline{S P L}_{i}$, the larger the topological persistence length of the correlation between $i$ and the rest of the network). In order to be consistent with the computation of the anatomical persistence length of correlation (i.e., the $A E D_{i}$ metric), also $\overline{S P L}_{i}$ was computed only in the aortic region from the inlet section to the first supraaortic trunk bifurcation point.

\section{Deciphering aortic hemodynamics - Canonical WSS-based hemodynamic descriptors}

In addition to the advanced methods for analyzing aortic hemodynamics introduced above, flow disturbances were evaluated here also in terms of canonical WSS-based descriptors (Table 1), namely the time-averaged wall shear stress (TAWSS), oscillatory shear index (OSI) [49], and relative residence time (RRT) [50]. Moreover, a descriptor of WSS multidirectionality was considered (Table 1), i.e., the transversal WSS (transWSS) [51], defined as the average WSS component acting orthogonal to the time-averaged WSS vector direction. 
271 To investigate the existence of possible relations between ATAA hemodynamics and the 272 mechanical properties of the aortic wall, an analysis of co-localization between exposure to 273 "disturbed" hemodynamics vs. wall stiffness was carried out according to schemes proposed 274 elsewhere $[28,29,52]$. More specifically, objective thresholds for WSS-based hemodynamic 275 descriptors and for wall stiffness were identified as the $2^{\text {nd }}$ tertile of the respective luminal 276 distribution ( $1^{\text {st }}$ tertile for TAWSS). The co-localization of luminal surface areas (SAs) characterized 277 by stiffness and each one of OSI, RRT, transWSS, or TSVI values higher (lower for TAWSS) than the 278 respective thresholds was quantitatively assessed by the similarity index (SI) [52]:

$279 \mathrm{SI}=\frac{2\left(\mathrm{SA}_{\text {highstiffness }} \cap \mathrm{SA}_{j}\right)}{\mathrm{SA}_{\text {highstiffness }}+\mathrm{SA}_{j}}$,

280 with $j$ indicating a generic WSS-based hemodynamic descriptor. SI ranges between 0 (the SAs have 281 no spatial overlap) and 1 (the SAs are equivalent and perfectly spatially overlapped). 


\section{Results}

ATAA wall stiffness in vivo estimation

The in vivo estimated local wall stiffness distribution on the AAo of the diseased subject is presented in Figure 3. Consistent with previous observations [53,54], local variations in the wall mechanical properties are evident in the investigated model, where the highest values of the stiffness are observed at the outer AAo wall. For this reason, in order to investigate the possible role played by hemodynamic features in the development and progression of aneurysm disease, the analysis was here focused at the proximal AAo outer wall of both healthy and diseased models, where the biomechanical wall alteration mainly occurred in the ATAA subject.

\section{WSS topological skeleton analysis}

As suggested elsewhere [12], here the analysis of the WSS vector field topological skeleton at the aortic luminal surface was applied in a two-step strategy.

As a first step, the aortic cycle-average WSS vector field topological skeleton was identified and analyzed (Figure 4a) aiming at identifying (on average) differences between the healthy and ATAA model. It emerged that on both models a line of marked WSS expansion was located at the proximal AAo outer wall, as identified by positive DIV values. However, the healthy aorta only presented there an unstable node. Moreover, marked contraction lines characterized the WSS topological skeleton on the ATAA outer wall, but they were not present on the luminal surface of the healthy aorta.

In the second step, the impact of the WSS vector field dynamics (along the cardiac cycle) on topological skeleton features was analyzed. The analysis of the luminal surface distribution of fixed points weighted residence time $\left(R T \nabla_{x_{f p}}\right.$, Figure $\left.4 \mathrm{~b}\right)$ highlighted the presence of a wide region at the AAo outer-wall of the diseased model where instantaneous WSS fixed points appear and reside for a longer fraction of cardiac cycle. Such region is surrounded by luminal regions with a 
high local WSS contraction/expansion strength. On the other hand, a completely different

distribution was observed on the outer wall of the healthy model (Figure 4b), mainly characterized by low $R T \nabla_{x_{f p}}$ values.

The analysis of the TSVI luminal distributions (Figure 4c) highlighted that: (1) the highest local variations in the contraction/expansion action exerted by the WSS on the endothelium along the cardiac cycle are located at two distinct regions of the AAo outer wall luminal surface, in both healthy and diseased models; (2) in the diseased model, high TSVI and high $R T \nabla_{x_{f p}}$ regions are markedly co-localized on the outer wall luminal surface.

\section{CNs analysis}

In the attempt to better describe differences between healthy and ATAA models accounting for possible links between the biomechanical alteration of the wall and hemodynamics, CNs were applied to the aortic flow and WSS fields.

The $D C$ distributions of the $V_{a x^{-}}, H_{k^{-}}$and $|\tau|$-based $C N s$ are displayed in Figure 5 for both models. For visualization purposes, in each CN the $D C$ values were classified in "high" and "low" based on the median value of the $D C$ distribution of the healthy subject. Regarding the axial flow in the AAo of the diseased model (Figure 5a), a predominance of $V_{a x}$ waveforms with a neighborhood which is representative of a portion of the whole aortic axial flow smaller than in the healthy model emerged, as highlighted by the low value $D C$ distribution in that region.

Regarding $H_{k}$, the $D C$ distribution in the AAo (Figure $5 b$ ): (1) confirms the presence of counterrotating helical flow patterns both in the healthy and in the ATAA model $[22,36,38,44]$, as suggested elsewhere [27]; (2) highlights that helical flow (characterized considering $H_{k}$ waveforms along the cardiac cycle) presents a spatiotemporal heterogeneity that in the ATAA model is markedly different from the healthy one, and which could be the consequence of the combination of the AAo dilatation and of the different shape of the (measured and imposed) inflow velocity 
profile (reminding that (1) the investigated ATAA subject is affected by BAV, and (2) that the presence of valvulopathy could markedly affect the AAo hemodynamics [55].

Figure 5 confirms that the differences in the spatiotemporal intravascular complexity characterizing the ATAA and healthy models reflect WSS magnitude waveforms heterogeneity. In detail, the distal segment of the AAo of the ATAA model is characterized by an extended region of the luminal surface of the dilated aorta exposed to $|\tau|$ waveforms presenting a neighborhood which is smaller than in the healthy model (low $D C$ values, Figure $5 c$ ). Therefore, in the diseased subject the pathologic expansion of the aortic wall where stiffness is higher (Figure 3 ) translates into WSS magnitude waveforms with a shape markedly different from the rest of the vessel. The impact of ATAA on the spatiotemporal hemodynamic heterogeneity in the AAo was evaluated also in terms of anatomical persistence length of the correlation between neighbor waveforms. AED maps (Figure 6a-b) highlight how the aortic dilation disrupts the persistence of the correlation of the $V_{a x}$ and $H_{k}$ waveforms, breaking the topological connections among waveforms in the AAo and shortening the length of correlation, compared to the healthy model. Finally, the heterogeneity of WSS magnitude spatiotemporal features induced by AAo dilatation, already emerged from the $D C$ maps, was confirmed by $\overline{S P L}$ maps. More specifically, it was observed that the luminal surface of the distal dilated AAo presents larger areas where the functional length of correlation of WSS magnitude waveforms is smaller than the healthy AAo (Figure 6c).

\section{Aortic hemodynamics vs. wall stiffness}

Here we assess the co-localization of the in vivo estimated wall stiffness at AAo outer wall of the ATAA model with the WSS topological skeleton-based quantities, and with canonical WSS-based descriptors of disturbed shear. A visual inspection of Figure 7 suggests that an association might exist between instantaneous WSS fixed points dynamics at the luminal surface and the aortic stiffness. Interestingly, instantaneous WSS fixed points undergo longer combinations of residence 
354 time and marked contraction/expansion strength of the WSS vector field (as expressed by the 355 quantity $R T \nabla_{x_{f p}}$ ) on those ATAA luminal surface areas where stiffness is high (Figure 7).

356 To explore more in depth the possible connections between peculiar WSS features and the altered 357 biomechanical properties of the diseased aortic wall, the extent of the co-localization of high 358 aortic wall stiffness regions with SAs exposed to presumably disturbed shear was quantified. By 359 visual inspection of Figure 8a, it emerged that high stiffness co-localizes with high TSVI better than 360 with canonical WSS-based descriptors. Of note, it was observed that SAs exposed to low TAWSS 361 poorly co-localize with high stiffness regions. These observations were quantitatively confirmed by 362 the SI values (Figure 8b), remarking the stronger capability of the WSS vector field divergence363 based quantity TSVI as indicator of altered arterial wall mechanical properties, than the canonical 364 WSS-based descriptors. Indeed, TSVI co-localizes with ATAA wall stiffness $206 \%$ more than TAWSS, $36515 \%$ more than OSI and transWSS, and 45\% more than RRT. 


\section{Discussion}

368

369

370

371

372

373

374

Wall dilatation/degeneration of the ascending aorta is a complex multifactorial process promoted by a unique bio-chemo-mechanical environment, which may ultimately lead to aortic wall dissection/rupture [7-13]. Despite a large body of literature focused on the role played by individual biological, chemical, or biomechanical factors in the ATAA development [12], the heterogeneity characterizing ATAA disease cannot be exhaustively explained by individual features and the analysis of their complex interplay is still poorly investigated $[10,12]$.

In this context, here we aim to highlight the possible links between ATAA hemodynamics, obtained by CFD simulations and explored with advanced methods, and in vivo estimated wall mechanical properties, inversely derived from gated CT scans [30].

The findings of this study confirm, on the basis of an in-depth quantitative exploration, that the near-wall and intravascular hemodynamic environment characterizing the ATAA model markedly differs from the healthy aorta.

More in detail, the analysis of the WSS topological skeleton highlighted peculiar features on the ATAA outer wall, which are not or are poorly present on the luminal surface of the healthy ascending aorta (Figure 4), i.e.: (1) the presence of localized marked WSS contraction regions; (2) the appearance and persistence of instantaneous WSS fixed points along the cardiac cycle (as quantified by $R T \nabla_{x_{f p}}$, which additionally weights the strength of the local contraction/expansion action exerted by the WSS); (3) a large luminal surface area undergoing marked variation in the WSS contraction/expansion action on the endothelium over the cardiac cycle (as indicated by TSVI).

From the CNs-based analysis, marked differences between ATAA and healthy aorta emerged in the organization of the intravascular hemodynamic patterns. In detail, here for the first time the disruption of spatiotemporal homogeneity of intravascular hemodynamic features induced by the 
AAo dilatation was quantified in terms of the Euclidian length of persistence of the correlation between flow quantities. As indicated by $A E D$, the presence of aortic dilation seems to reduce the anatomical length of correlation persistence both for the axial velocity and kinetic helicity density waveforms, compared to the healthy case (Figure 6).

\section{This induced-by-aortic-dilation increase in AAo intravascular hemodynamics spatiotemporal} heterogeneity reflects on a reduced functional length of correlation in WSS magnitude waveforms , localized on the distal outer wall, as indicated by $\overline{S P L}$ (Figure 6). Such differences between ATAA and healthy models are further confirmed by the observed WSS magnitude $D C$ luminal distribution, which highlights that in the dilated aorta the topologically isolated region is more extended than in the healthy one (Figure $5 \mathrm{c}$ ). The latter result can be explained by the demonstrated causative role of helical flow in determining WSS patterns at the aortic luminal surface $[28,29]$.

The evidences emerged from the WSS topological skeleton analysis and from the CNs-based analysis support the hypothesis that a complex interplay exists between hemodynamic features, aortic wall dilatation and its degradation in terms of wall mechanical properties. This statement is supported by the main finding of the study, i.e., that in the distal AAo outer wall of the ATAA model, extended high stiffness regions (indicating a degradation of the mechanical properties of the wall), co-localize with luminal surface areas of: (1) high instantaneous WSS fixed points residence time (weighted by WSS divergence value) (Figure 7); (2) large variations in the WSS contraction/expansion action exerted by the flowing blood along the cardiac cycle, here quantified by TSVI (Figure 8).

It is worth noting that the cross-sectional nature of this study does not allow to establish whether the observed hemodynamic features are responsible for wall dilatation and degradation, or whether they are consequence of the dilated geometry. However, previous studies suggested that 
415 altered aortic flow predisposes the ascending thoracic aorta to wall dilatation/degradation $[21,23]$

416 as a consequence of the purported role of endothelial shear in the pathogenesis of ATAA [56]. In

417 this sense, the findings of this study suggest that future studies addressing the role of

418 hemodynamics in the ascending thoracic aorta to wall dilatation/degradation should include

419 descriptors based on the WSS topological skeleton, such as TSVI (Figure 8). In fact, such descriptors

420 show a higher co-localization with regions at the aortic wall characterized by high stiffness than

421 canonical WSS-based descriptors. The hemodynamic quantities presented here may improve the

422 prediction of ATAA development and progression and contribute to a deeper understanding of the

423 underlying hemodynamics-driven processes.

424

425

\section{Limitations}

Several limitations could weaken the findings of this study. The main limitation is that the present analysis is based on only one ATAA model and one healthy aorta model. For this reason, we acknowledge that the here reported association between the WSS topological skeleton-based descriptor TSVI and ATAA wall weakening indicator should be confirmed on a larger dataset.

430 The inverse approach here adopted to estimate local wall stiffness in vivo is based on the 431 assumption that the aortic wall behaves as a membrane with no through-thickness shear $[30,57]$.

432 The regions near the supra-aortic branches may not satisfy such assumption and were therefore excluded from the wall stiffness analysis. Moreover, in the perspective of extending the analysis of aortic stiffness at different stages of ATAA growth, an extensive use of the inverse approach may

435 be limited by the use of X-ray during the gated CT scans procedure. In this regard, other 436 techniques, such as ultrasounds [58] or MRI [59], may be adopted as a source of dynamic images 437 of the aortic wall during the cardiac cycle. 
438 Finally, the main limitation regarding the numerical settings of the CFD study is represented by the

439 rigid wall assumption. However, recent observations have demonstrated that aortic wall motion 440 has a minor impact on WSS-based descriptors of disturbed shear [60], and on intravascular flow 441 topology. Concerning the latter, for example, similar helical fluid structures have been observed in 442 vivo $[38,44,61]$ and in vitro both in rigid and distensible aortic phantoms $[62,63]$. Therefore, we 443 believe that the assumption on aortic wall distensibility does not entail the generality of the 444 present results. 
445 Conclusions

446 In this study, the integration of computational hemodynamics, in vivo stiffness estimation and 447 advanced fluid mechanics methods allowed to explore more in depth the existence of links 448 between the altered hemodynamics in the ATAA and the wall biomechanical degradation.

449 Moreover, combining WSS topological skeleton analysis and CNs-based analysis has highlighted 450 that WSS topological skeleton features across the luminal surface of the ascending aorta are 451 markedly affected by the different level of spatiotemporal heterogeneity characterizing the ATAA 452 and healthy subjects' hemodynamics.

453 In conclusion, despite the exploratory nature of the study, advanced methods of analysis of the 454 aortic blood flow have shown their very strong potential to decipher the links between local 455 hemodynamics and the degradation of the mechanical properties of the aortic wall. In this sense, 456 WSS topological skeleton analysis $[25,26]$ and CNs-based analysis promise to be very effective 457 tools $[25,26]$. 


\section{References}

460 [1] Isselbacher EM. Thoracic and abdominal aortic aneurysms. Circulation 2005;111:816-28.

[2] Erbel R, Aboyans V, Boileau C, Bossone E, Bartolomeo R Di, Eggebrecht H, et al. 2014 ESC Guidelines on the diagnosis and treatment of aortic diseases: Document covering acute and chronic aortic diseases of the thoracic and abdominal aorta of the adult. The Task Force for the Diagnosis and Treatment of Aortic Diseases of the European . Eur Heart J 2014;35:2873926. https://doi.org/10.1093/eurheartj/ehu281.

[3] Coady MA, Rizzo JA, Hammond GL, Kopf GS, Elefteriades JA. Surgical intervention criteria for thoracic aortic aneurysms: a study of growth rates and complications. Ann Thorac Surg 1999;67:1922-8. https://doi.org/10.1016/s0003-4975(99)00431-2.

[4] Johansson G, Markstrom U, Swedenborg J. Ruptured thoracic aortic aneurysms: a study of incidence and mortality rates. J Vasc Surg 1995;21:985-8. https://doi.org/10.1016/s07415214(95)70227-x.

[5] Pape LA, Tsai TT, Isselbacher EM, Oh JK, O'gara PT, Evangelista A, et al. Aortic diameter >or $=5.5 \mathrm{~cm}$ is not a good predictor of type $A$ aortic dissection: observations from the International Registry of Acute Aortic Dissection (IRAD). Circulation 2007;116:1120-7. https://doi.org/10.1161/CIRCULATIONAHA.107.702720.

[6] Chau KH, Elefteriades JA. Natural history of thoracic aortic aneurysms: size matters, plus moving beyond size. Prog Cardiovasc Dis 2013;56:74-80. https://doi.org/10.1016/j.pcad.2013.05.007.

[7] Elefteriades JA, Farkas EA. Thoracic aortic aneurysm clinically pertinent controversies and uncertainties. J Am Coll Cardiol 2010;55:841-57. https://doi.org/10.1016/j.jacc.2009.08.084.

[8] Poullis MP, Warwick R, Oo A, Poole RJ. Ascending aortic curvature as an independent risk factor for type $A$ dissection, and ascending aortic aneurysm formation: a mathematical model. Eur J Cardiothorac Surg 2008;33:995-1001.

https://doi.org/10.1016/j.ejcts.2008.02.029.

[9] Gallo A, Agnese V, Coronnello C, Raffa GM, Bellavia D, Conaldi PG, et al. On the prospect of serum exosomal miRNA profiling and protein biomarkers for the diagnosis of ascending aortic dilatation in patients with bicuspid and tricuspid aortic valve. Int J Cardiol 2018;273:230-6. https://doi.org/https://doi.org/10.1016/j.ijcard.2018.10.005.

[10] Agnese V, Pasta S, Michelena HI, Minà C, Romano GM, Carerj S, et al. Patterns of ascending aortic dilatation and predictors of surgical replacement of the aorta: A comparison of bicuspid and tricuspid aortic valve patients over eight years of follow-up. J Mol Cell Cardiol 2019;135:31-9. https://doi.org/https://doi.org/10.1016/j.yjmcc.2019.07.010.

[11] Pasta S, Agnese V, Di Giuseppe M, Gentile G, Raffa GM, Bellavia D, et al. In Vivo Strain Analysis of Dilated Ascending Thoracic Aorta by ECG-Gated CT Angiographic Imaging. Ann Biomed Eng 2017;45:2911-20. https://doi.org/10.1007/s10439-017-1915-4. 
[12] Wagenseil JE. Bio-chemo-mechanics of thoracic aortic aneurysms. Curr Opin Biomed Eng 2018;5:50-7. https://doi.org/10.1016/J.COBME.2018.01.002.

[13] Petit C, Mousavi SJ, Avril S. Review of the Essential Roles of SMCs in ATAA Biomechanics. Adv Biomech Tissue Regen 2019:95-114. https://doi.org/10.1016/B978-0-12-8163900.00006-6.

[14] Bollache E, Guzzardi DG, Sattari S, Olsen KE, Di Martino ES, Malaisrie SC, et al. Aortic valvemediated wall shear stress is heterogeneous and predicts regional aortic elastic fiber thinning in bicuspid aortic valve-associated aortopathy. J Thorac Cardiovasc Surg 2018;156:2112-2120.e2. https://doi.org/https://doi.org/10.1016/j.jtcvs.2018.05.095.

[15] Suwa K, Rahman OA, Bollache E, Rose MJ, Rahsepar AA, Carr JC, et al. Effect of Aortic Valve Disease on 3D Hemodynamics in Patients With Aortic Dilation and Trileaflet Aortic Valve Morphology. J Magn Reson Imaging 2020;51:481-91. https://doi.org/10.1002/jmri.26804.

[16] Dux-Santoy L, Guala A, Teixidó-Turà G, Ruiz-Muñoz A, Maldonado G, Villalva N, et al. Increased rotational flow in the proximal aortic arch is associated with its dilation in bicuspid aortic valve disease. Eur Hear J - Cardiovasc Imaging 2019;20:1407-17. https://doi.org/10.1093/ehjci/jez046.

[17] Rodríguez-Palomares JF, Dux-Santoy L, Guala A, Kale R, Maldonado G, Teixidó-Turà G, et al. Aortic flow patterns and wall shear stress maps by 4D-flow cardiovascular magnetic resonance in the assessment of aortic dilatation in bicuspid aortic valve disease. $J$ Cardiovasc Magn Reson 2018;20:28. https://doi.org/10.1186/s12968-018-0451-1.

[18] Youssefi P, Gomez A, Arthurs C, Sharma R, Jahangiri M, Alberto Figueroa C. Impact of Patient-Specific Inflow Velocity Profile on Hemodynamics of the Thoracic Aorta. J Biomech Eng 2017;140. https://doi.org/10.1115/1.4037857.

[19] Pasta S, Gentile G, Raffa GM, Bellavia D, Chiarello G, Liotta R, et al. In Silico Shear and Intramural Stresses are Linked to Aortic Valve Morphology in Dilated Ascending Aorta. Eur J Vasc Endovasc Surg 2017;54:254-63. https://doi.org/https://doi.org/10.1016/j.ejvs.2017.05.016.

[20] Mendez V, Di Giuseppe M, Pasta S. Comparison of hemodynamic and structural indices of ascending thoracic aortic aneurysm as predicted by 2-way FSI, CFD rigid wall simulation and patient-specific displacement-based FEA. Comput Biol Med 2018;100:221-9. https://doi.org/https://doi.org/10.1016/j.compbiomed.2018.07.013.

[21] Condemi F, Campisi S, Viallon M, Croisille P, Fuzelier J-F, Avril S. Ascending thoracic aorta aneurysm repair induces positive hemodynamic outcomes in a patient with unchanged bicuspid aortic valve. J Biomech 2018;81:145-8. https://doi.org/10.1016/j.jbiomech.2018.09.022.

[22] Condemi F, Campisi S, Viallon M, Troalen T, Xuexin G, Barker AJ, et al. Fluid- and Biomechanical Analysis of Ascending Thoracic Aorta Aneurysm with Concomitant Aortic Insufficiency. Ann Biomed Eng 2017;45:2921-32. https://doi.org/10.1007/s10439-0171913-6.

[23] Burk J, Blanke P, Stankovic Z, Barker A, Russe M, Geiger J, et al. Evaluation of 3D blood flow 
patterns and wall shear stress in the normal and dilated thoracic aorta using flow-sensitive 4D CMR. J Cardiovasc Magn Reson 2012;14:84. https://doi.org/10.1186/1532-429X-14-84.

[24] Guzzardi DG, Barker AJ, van Ooij P, Malaisrie SC, Puthumana JJ, Belke DD, et al. ValveRelated Hemodynamics Mediate Human Bicuspid Aortopathy: Insights From Wall Shear Stress Mapping. J Am Coll Cardiol 2015;66:892-900. https://doi.org/10.1016/j.jacc.2015.06.1310.

[25] Mazzi V, Gallo D, Calo K, Najafi M, Khan MO, De Nisco G, et al. A Eulerian method to analyze wall shear stress fixed points and manifolds in cardiovascular flows. Biomech Model Mechanobiol 2019. https://doi.org/10.1007/s10237-019-01278-3.

[26] Arzani A, Shadden SC. Wall shear stress fixed points in cardiovascular fluid mechanics. J Biomech 2018;73:145-52. https://doi.org/10.1016/j.jbiomech.2018.03.034.

[27] Calo K, Gallo D, Steinman DA, Mazzi V, Scarsoglio S, Ridolfi L, et al. Spatiotemporal Hemodynamic Complexity in Carotid Arteries: an Integrated Computational Hemodynamics \& Complex Networks-Based Approach. IEEE Trans Biomed Eng 2019. https://doi.org/10.1109/TBME.2019.2949148.

[28] De Nisco G, Kok AM, Chiastra C, Gallo D, Hoogendoorn A, Migliavacca F, et al. The Atheroprotective Nature of Helical Flow in Coronary Arteries. Ann Biomed Eng 2019;47:425-38. https://doi.org/10.1007/s10439-018-02169-x.

[29] De Nisco G, Hoogendoorn A, Chiastra C, Gallo D, Kok AM, Morbiducci U, et al. The impact of helical flow on coronary atherosclerotic plaque development. Atherosclerosis 2020. https://doi.org/10.1016/J.ATHEROSCLEROSIS.2020.01.027.

[30] Farzaneh S, Trabelsi O, Avril S. Inverse identification of local stiffness across ascending thoracic aortic aneurysms. Biomech Model Mechanobiol 2019;18:137-53. https://doi.org/10.1007/s10237-018-1073-0.

[31] Dillon-Murphy D, Noorani A, Nordsletten D, Figueroa CA. Multi-modality image-based computational analysis of haemodynamics in aortic dissection. Biomech Model Mechanobiol 2016;15:857-76. https://doi.org/10.1007/s10237-015-0729-2.

[32] Updegrove A, Wilson NM, Merkow J, Lan H, Marsden AL, Shadden SC. SimVascular: An Open Source Pipeline for Cardiovascular Simulation. Ann Biomed Eng 2017;45:525-41. https://doi.org/10.1007/s10439-016-1762-8.

[33] Morbiducci U, Ponzini R, Gallo D, Bignardi C, Rizzo G. Inflow boundary conditions for imagebased computational hemodynamics: impact of idealized versus measured velocity profiles in the human aorta. J Biomech 2013;46:102-9. https://doi.org/10.1016/j.jbiomech.2012.10.012.

[34] Gallo D, De Santis G, Negri F, Tresoldi D, Ponzini R, Massai D, et al. On the use of in vivo measured flow rates as boundary conditions for image-based hemodynamic models of the human aorta: implications for indicators of abnormal flow. Ann Biomed Eng 2012;40:72941. https://doi.org/10.1007/s10439-011-0431-1.

[35] Calò K, De Nisco G, Gallo D, Chiastra C, Hoogendoorn A, Steinman DA, et al. Exploring wall shear stress spatiotemporal heterogeneity in coronary arteries combining correlation-based 
analysis and complex networks with computational hemodynamics. Proc Inst Mech Eng $\mathrm{H}$ 2020:(under review).

[36] Morbiducci U, Gallo D, Cristofanelli S, Ponzini R, Deriu MA, Rizzo G, et al. A rational approach to defining principal axes of multidirectional wall shear stress in realistic vascular geometries, with application to the study of the influence of helical flow on wall shear stress directionality in aorta. J Biomech 2015;48:899-906. https://doi.org/10.1016/j.jbiomech.2015.02.027.

[37] Gallo D, B. BP, Morbiducci U, Ye Q, (Joyce) XY, Maryam E, et al. Segment-specific associations between local haemodynamic and imaging markers of early atherosclerosis at the carotid artery: an in vivo human study. J R Soc Interface 2018;15:20180352. https://doi.org/10.1098/rsif.2018.0352.

[38] Morbiducci U, Ponzini R, Rizzo G, Cadioli M, Esposito A, De Cobelli F, et al. In vivo quantification of helical blood flow in human aorta by time-resolved three-dimensional cine phase contrast magnetic resonance imaging. Ann Biomed Eng 2009;37:516-31. https://doi.org/10.1007/s10439-008-9609-6.

[39] Liu X, Sun A, Fan Y, Deng X. Physiological significance of helical flow in the arterial system and its potential clinical applications. Ann Biomed Eng 2015;43:3-15. https://doi.org/10.1007/s10439-014-1097-2.

[40] Stonebridge PA, Suttie SA, Ross R, Dick J. Spiral Laminar Flow: a Survey of a ThreeDimensional Arterial Flow Pattern in a Group of Volunteers. Eur J Vasc Endovasc Surg 2016;52:674-80. https://doi.org/10.1016/j.ejvs.2016.07.018.

[41] Gallo D, Steinman DA, Bijari PB, Morbiducci U. Helical flow in carotid bifurcation as surrogate marker of exposure to disturbed shear. J Biomech 2012;45:2398-404. https://doi.org/10.1016/j.jbiomech.2012.07.007.

[42] Gallo D, Steinman DA, Morbiducci U. An insight into the mechanistic role of the common carotid artery on the hemodynamics at the carotid bifurcation. Ann Biomed Eng 2015;43:68-81. https://doi.org/10.1007/s10439-014-1119-0.

[43] Morbiducci U, Ponzini R, Grigioni M, Redaelli A. Helical flow as fluid dynamic signature for atherogenesis risk in aortocoronary bypass. A numeric study. J Biomech 2007;40:519-34. https://doi.org/10.1016/j.jbiomech.2006.02.017.

[44] Morbiducci U, Ponzini R, Rizzo G, Cadioli M, Esposito A, Montevecchi FM, et al. Mechanistic insight into the physiological relevance of helical blood flow in the human aorta: an in vivo study. Biomech Model Mechanobiol 2011;10:339-55. https://doi.org/10.1007/s10237-0100238-2.

[45] De Nisco G, Kok AM, Chiastra C, Gallo D, Hoogendoorn A, Migliavacca F, et al. The Atheroprotective Nature of Helical Flow in Coronary Arteries. Ann Biomed Eng 2019;47. https://doi.org/10.1007/s10439-018-02169-x.

[46] Kilner PJ, Yang GZ, Mohiaddin RH, Firmin DN, Longmore DB. Helical and retrograde secondary flow patterns in the aortic arch studied by three-directional magnetic resonance velocity mapping. Circulation 1993;88:2235-47. https://doi.org/10.1161/01.cir.88.5.2235. 
[47] Scarsoglio S, lacobello G, Ridolfi L. Complex networks unveiling spatial patterns in turbulence. Int J Bifurc Chaos 2016;26:1-12. https://doi.org/10.1142/S0218127416502230.

[48] Boccaletti S, Latora V, Moreno Y, Chavez M, Hwang DU. Complex networks: Structure and dynamics. Phys Rep 2006;424:175-308. https://doi.org/10.1016/j.physrep.2005.10.009.

[49] Ku DN, Giddens DP, Zarins CK, Glagov S. Pulsatile flow and atherosclerosis in the human carotid bifurcation. Positive correlation between plaque location and low oscillating shear stress. Arteriosclerosis 1985;5:293-302. https://doi.org/10.1161/01.atv.5.3.293.

[50] Himburg HA, Grzybowski DM, Hazel AL, LaMack JA, Li X-M, Friedman MH. Spatial comparison between wall shear stress measures and porcine arterial endothelial permeability. Am J Physiol Heart Circ Physiol 2004;286:H1916-22. https://doi.org/10.1152/ajpheart.00897.2003.

[51] Peiffer V, Sherwin SJ, Weinberg PD. Computation in the rabbit aorta of a new metric - the transverse wall shear stress - to quantify the multidirectional character of disturbed blood flow. J Biomech 2013;46:2651-8. https://doi.org/10.1016/j.jbiomech.2013.08.003.

[52] Gallo D, Steinman DA, Morbiducci U. Insights into the co-localization of magnitude-based versus direction-based indicators of disturbed shear at the carotid bifurcation. J Biomech 2016;49:2413-9. https://doi.org/10.1016/j.jbiomech.2016.02.010.

[53] Choudhury N, Bouchot O, Rouleau L, Tremblay D, Cartier R, Butany J, et al. Local mechanical and structural properties of healthy and diseased human ascending aorta tissue. Cardiovasc Pathol 2009;18:83-91. https://doi.org/10.1016/j.carpath.2008.01.001.

[54] Khanafer K, Duprey A, Zainal M, Schlicht M, Williams D, Berguer R. Determination of the elastic modulus of ascending thoracic aortic aneurysm at different ranges of pressure using uniaxial tensile testing. J Thorac Cardiovasc Surg 2011;142:682-6. https://doi.org/10.1016/j.jtcvs.2010.09.068.

[55] Pirola S, Jarral OA, O’Regan DP, Asimakopoulos G, Anderson JR, Pepper JR, et al. Computational study of aortic hemodynamics for patients with an abnormal aortic valve: The importance of secondary flow at the ascending aorta inlet. APL Bioeng 2018;2:26101. https://doi.org/10.1063/1.5011960.

[56] Michel JB, Jondeau G, Milewicz DiM. From genetics to response to injury: Vascular smooth muscle cells in aneurysms and dissections of the ascending aorta. Cardiovasc Res 2018;114:578-89. https://doi.org/10.1093/cvr/cvy006.

[57] Farzaneh S, Trabelsi O, Chavent B, Avril S. Identifying Local Arterial Stiffness to Assess the Risk of Rupture of Ascending Thoracic Aortic Aneurysms. Ann Biomed Eng 2019;47:1038-50. https://doi.org/10.1007/s10439-019-02204-5.

[58] Wittek A, Karatolios K, Fritzen C-P, Bereiter-Hahn J, Schieffer B, Moosdorf R, et al. Cyclic three-dimensional wall motion of the human ascending and abdominal aorta characterized by time-resolved three-dimensional ultrasound speckle tracking. Biomech Model Mechanobiol 2016;15:1375-88. https://doi.org/10.1007/s10237-016-0769-2.

[59] Arani A, Arunachalam SP, Chang ICY, Baffour F, Rossman PJ, Glaser KJ, et al. Cardiac MR elastography for quantitative assessment of elevated myocardial stiffness in cardiac 
amyloidosis. J Magn Reson Imaging 2017;46:1361-7. https://doi.org/10.1002/jmri.25678.

[60] Lantz J, Renner J, Karlsson M. Wall shear stress in a subject specific human aorta - Influence of fluid-structure interaction. Int J Appl Mech 2011;03:759-78. https://doi.org/10.1142/S1758825111001226.

[61] Frydrychowicz A, Berger A, Munoz Del Rio A, Russe MF, Bock J, Harloff A, et al. Interdependencies of aortic arch secondary flow patterns, geometry, and age analysed by 4dimensional phase contrast magnetic resonance imaging at 3 Tesla. Eur Radiol 2012;22:1122-30. https://doi.org/10.1007/s00330-011-2353-6.

[62] Gallo D, Gülan U, Di Stefano A, Ponzini R, Lüthi B, Holzner M, et al. Analysis of thoracic aorta hemodynamics using 3D particle tracking velocimetry and computational fluid dynamics. J Biomech 2014;47:3149-55. https://doi.org/https://doi.org/10.1016/j.jbiomech.2014.06.017.

[63] Gulan U, Luthi B, Holzner M, Liberzon A, Tsinober A, Kinzelbach W. Experimental Investigation of the Influence of the Aortic Stiffness on Hemodynamics in the Ascending Aorta. IEEE J Biomed Heal Informatics 2014;18:1775-80. https://doi.org/10.1109/JBHI.2014.2322934. 
675 Tables

676 Table 1: Definition of WSS-based hemodynamic descriptors of "disturbed flow".

WSS-based hemodynamic descriptors

Time Average WSS (TAWSS) TAWSS $=\frac{1}{T} \int_{0}^{T}|\boldsymbol{\tau}| d t$

Oscillatory Shear Index (OSI) $\mathrm{OSI}=0.5\left[1-\left(\frac{\left|\int_{0}^{T} \mathbf{\tau} d t\right|}{\int_{0}^{T}|\mathbf{\tau}| d t}\right)\right]$

Relative Residence Time (RRT) $\mathrm{RRT}=\frac{1}{\mathrm{TAWSS} \cdot(1-2 \cdot \mathrm{OSI})}=\frac{1}{\frac{1}{T}\left|\int_{0}^{T} \boldsymbol{\tau} d t\right|}$

Transversal WSS (transWSS) $\operatorname{transWSS}=\frac{1}{T} \int_{0}^{T}\left|\boldsymbol{\tau} \cdot\left(\mathbf{n} \times \frac{\int_{0}^{T} \boldsymbol{\tau} d t}{\left|\int_{0}^{T} \boldsymbol{\tau} d t\right|}\right)\right| d t$

$\mathbf{\tau}$ is the WSS vector; $T$ is the period of the cardiac cycle; $\mathbf{n}$ is the unit vector normal to the arterial 678 surface at each element.

679 


\section{Figures captions}

681 Figure 1. Schematics of the study. The diagram shows how imaging data contribute to define vessel 682 geometry, hemodynamic variables and wall mechanical properties. BCs: boundary conditions; CFD: computational fluid dynamics; ATAA: ascending thoracic aorta aneurysm; CNs: complex networks.

684 685 686

Figure 2. WSS topological skeleton features. (a) Configuration of each fixed point-type and contraction and expansion regions, colored by blue and red respectively. (b) Identification and classification of fixed points based on Poincaré index and eigenvalues of the Jacobian matrix.

Figure 3. Estimated wall stiffness distribution at the luminal surface of ATAA model.

Figure 4. Luminal distributions of WSS topological skeleton-based metrics. Aortic luminal distributions of (a) cycle-average WSS topological skeleton, (b) WSS fixed points weighted residence time $\left(R T \nabla_{x_{f p}}\right)$, and (c) TSVI. In panel (a), contraction/expansion regions are colored by blue/red, respectively, and Line Integral Convolution technique is adopted to visualize WSS vector. The ATAA and healthy aortic models are displayed in the top and bottom panel, respectively.

Figure 5. $D C$ distributions of hemodynamic descriptors-based CNs. The $D C$ distributions of the (a) $V_{a x}$ (b) $H_{k}$, and (c) $|\tau| C N s$ are displayed for the diseased (top) and healthy (bottom) subjects.

Figure 6. Anatomical and topological length of correlation persistence of hemodynamic spatiotemporal patterns. The $A E D$ distributions of the (a) $V_{a x}$, and (b) $H_{k} C N s$, and (c) $\overline{S P L}$ distribution of the $|\tau| C N s$ are displayed for the diseased (top) and healthy (bottom) subjects. $A E D$ and $\overline{S P L}$ were computed at the ascending aorta segment of each model.

Figure 7. Wall stiffness vs. weighted residence time analysis. Contour maps of estimated wall stiffness (left) and WSS fixed points weighted residence time $\left(R T \nabla_{x_{f p}}\right.$ - right) at the luminal surface of ATAA model. The black contour line represents the $2^{\text {nd }}$ tertile of stiffness luminal distribution at the ATAA outer wall.

Figure 8. Wall stiffness vs. deranged aortic hemodynamics analysis. (a) co-localization maps of high stiffness and disturbed shear; (b) similarity indices quantifying the co-localization of luminal SAs characterized by high stiffness and each descriptor of deranged hemodynamics (i.e., low TAWSS; high OSI, RRT, transWSS, TSVI). 
medical imaging

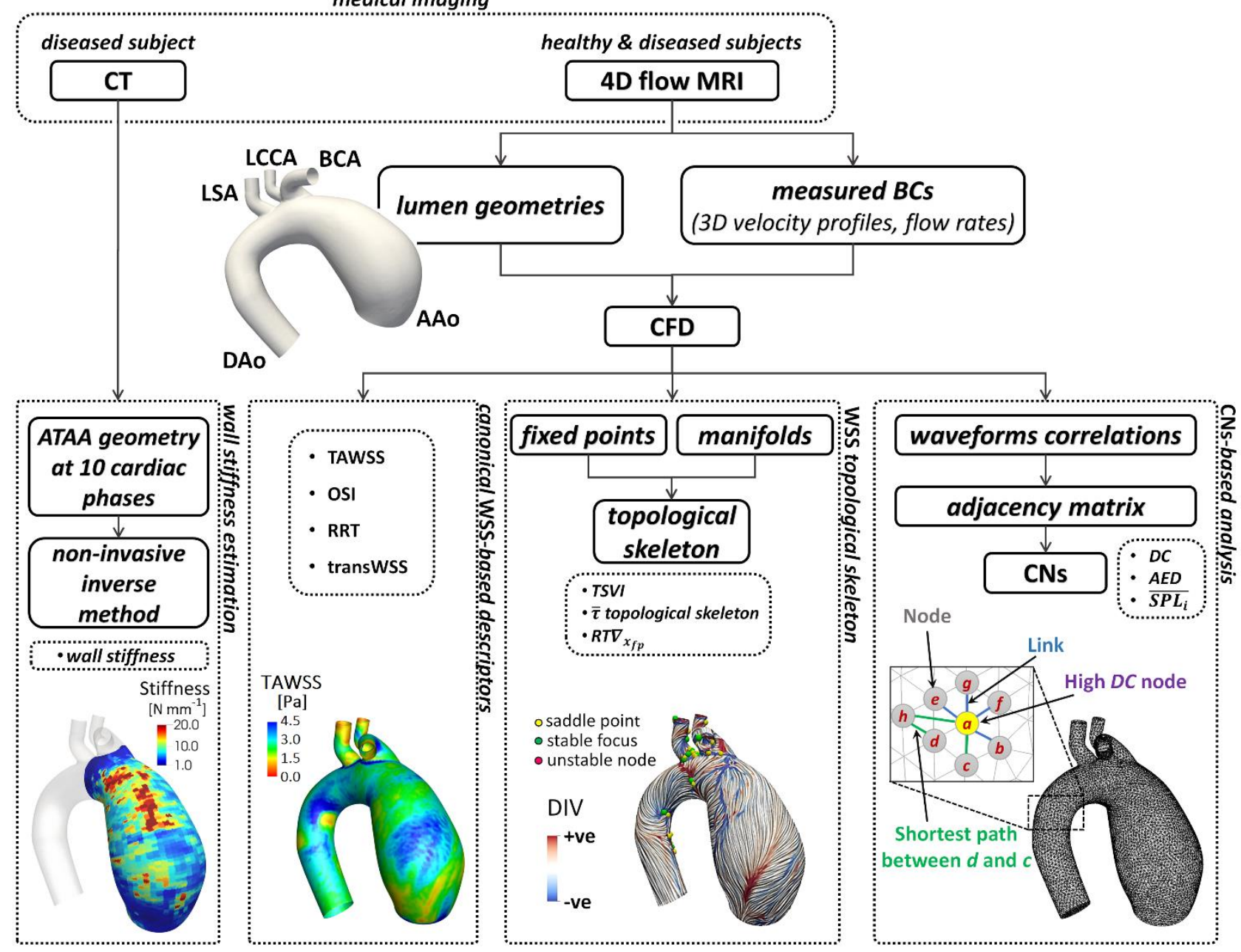

Figure 1. Schematics of the study. The diagram shows how imaging data contribute to define vessel geometry, hemodynamic variables and wall mechanical properties. BCs: boundary conditions; CFD: computational fluid dynamics; ATAA: ascending thoracic aorta aneurysm; CNs: complex networks. 


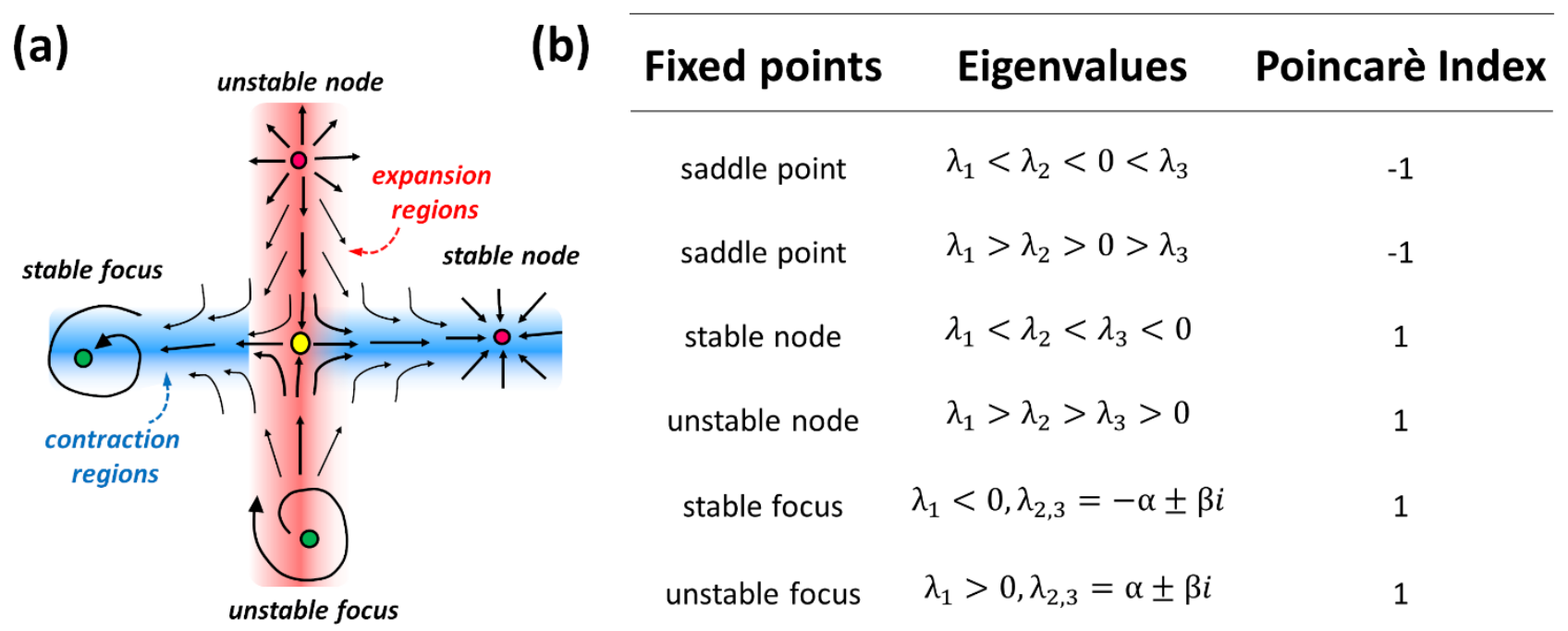

716

717

718

719

720

721

722

723

724

725

726

727

728

Figure 2. WSS topological skeleton features. (a) Configuration of each fixed point-type and contraction and expansion regions, colored by blue and red respectively. (b) Identification and classification of fixed points based on Poincaré index and eigenvalues of the Jacobian matrix.

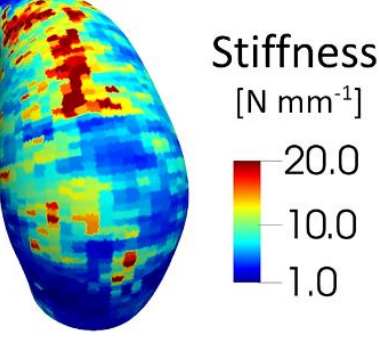

Figure 3. Estimated wall stiffness distribution at the luminal surface of ATAA model. 

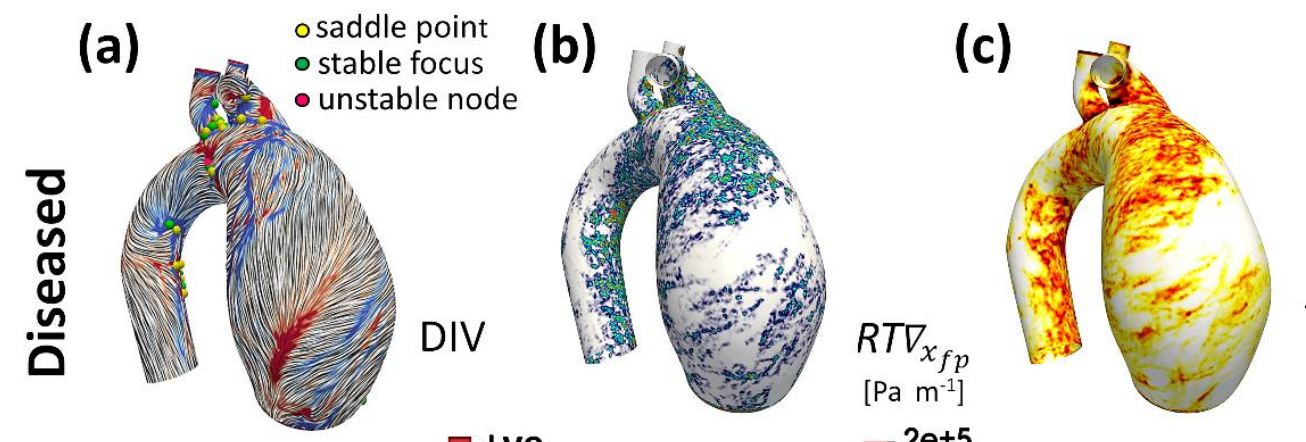

TSVI

$\left[\mathrm{m}^{-1}\right]$
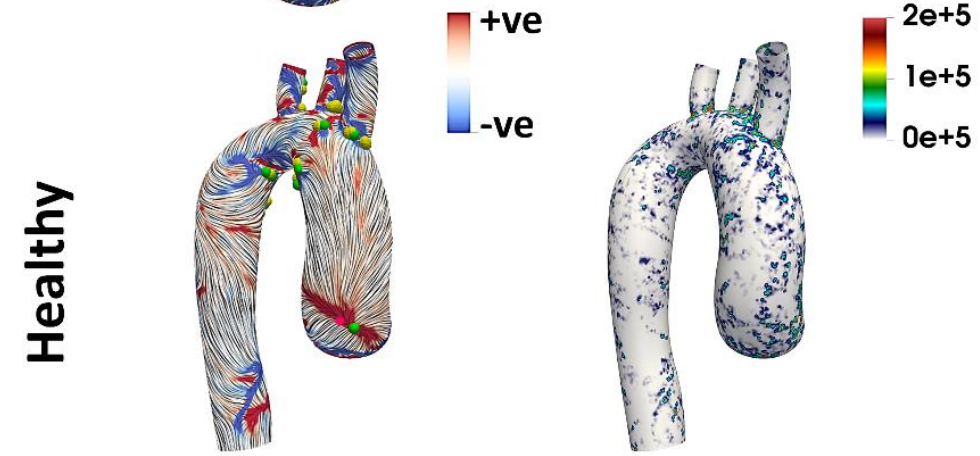

0.8
0.6
0.4
0.2

729

Figure 4. Luminal distributions of WSS topological skeleton-based metrics. Aortic luminal distributions of (a) cycle-average WSS topological skeleton, (b) WSS fixed points weighted residence time $\left(R T \nabla_{x_{f p}}\right)$, and (c) TSVI. In panel (a), contraction/expansion regions are colored by blue/red, respectively, and Line Integral Convolution technique is adopted to visualize WSS vector. The ATAA and healthy aortic models are displayed in the top and bottom panel, respectively.

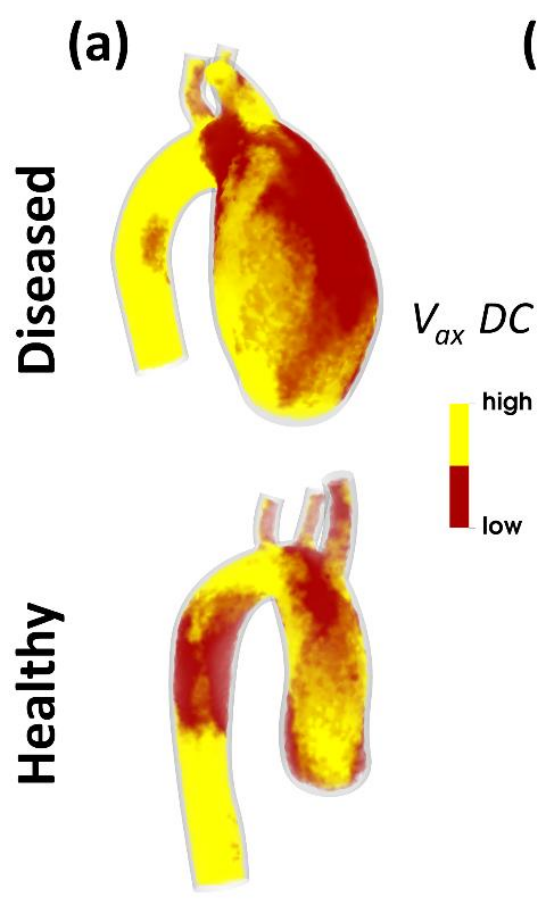

(b)

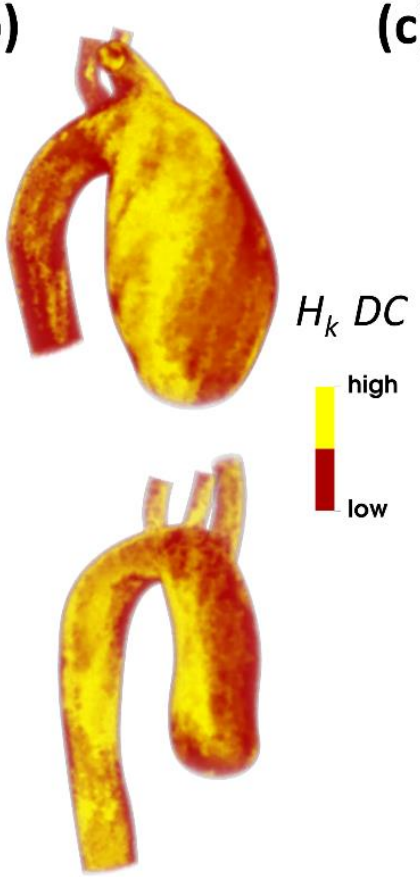

(c)
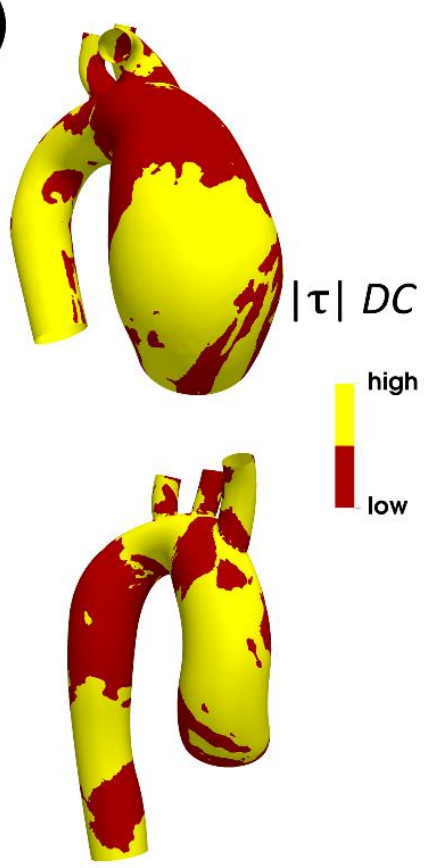

Figure 5. $D C$ distributions of hemodynamic descriptors-based CNs. The $D C$ distributions of the (a) $V_{a x}$ (b) $H_{k}$, and (c) $|\tau| C N s$ are displayed for the diseased (top) and healthy (bottom) subjects. 


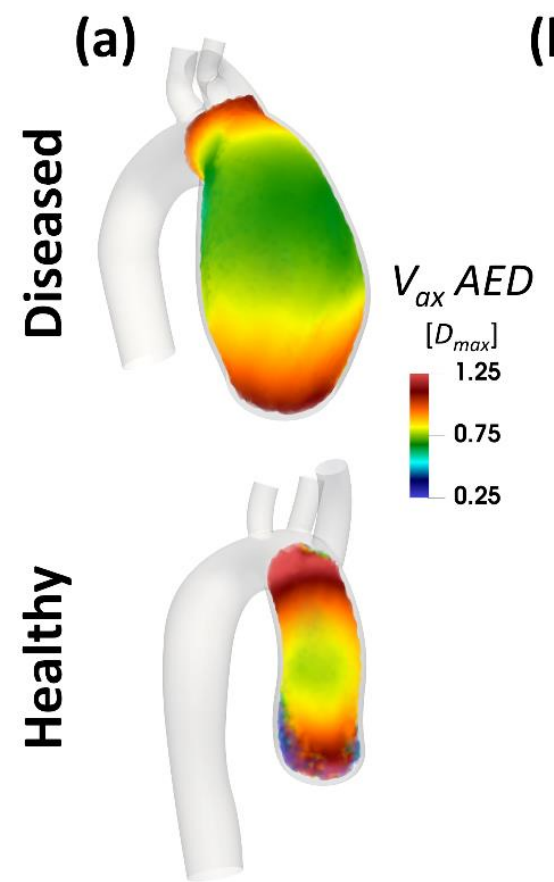

(b)

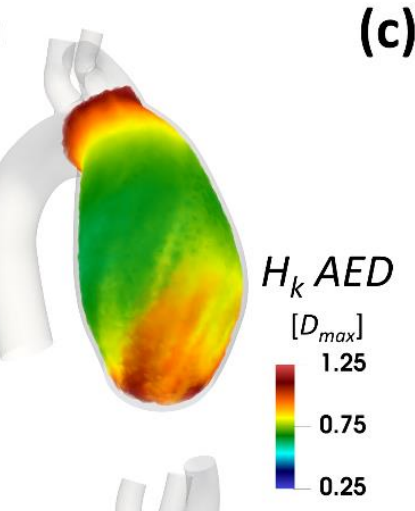

(c)

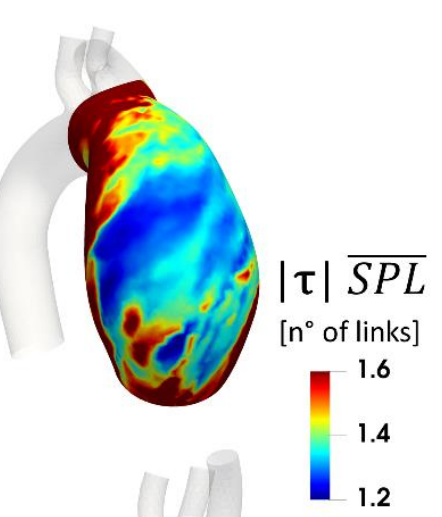

Figure 7. Wall stiffness vs. weighted residence time analysis. Contour maps of estimated wall stiffness (left) and WSS fixed points weighted residence time $\left(R T \nabla_{x_{f p}}\right.$ - right) at the luminal surface of ATAA model. The black contour line represents the $2^{\text {nd }}$ tertile of stiffness luminal distribution at the ATAA outer wall.

Figure 6. Anatomical and topological length of correlation persistence of hemodynamic spatiotemporal patterns. The $A E D$ distributions of the (a) $V_{a x}$, and (b) $H_{k} \mathrm{CNs}$, and (c) $\overline{S P L}$ distribution of the $|\tau| \mathrm{CNs}$ are displayed for the diseased (top) and healthy (bottom) subjects. AED and $\overline{S P L}$ were computed at the ascending aorta segment of each model.
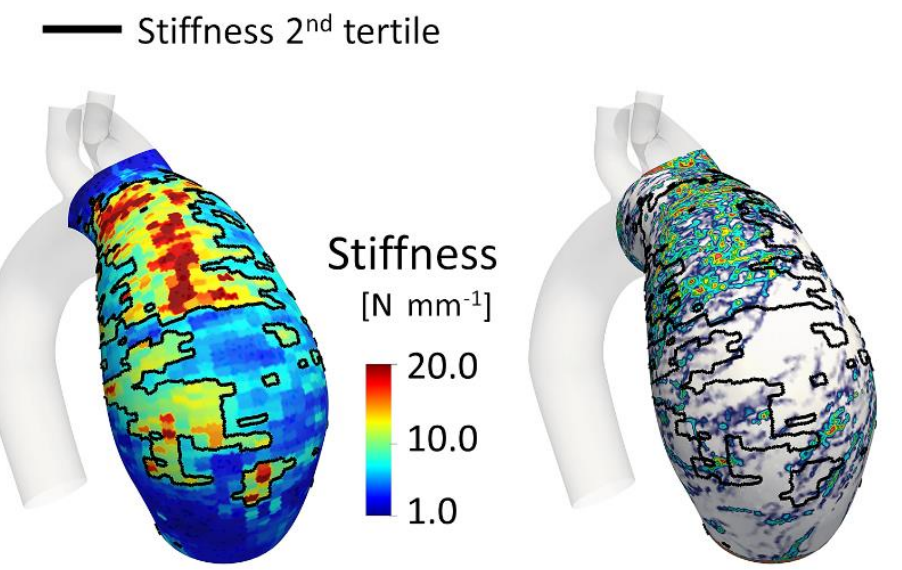

$0 \mathrm{e}+5$ 
(a)
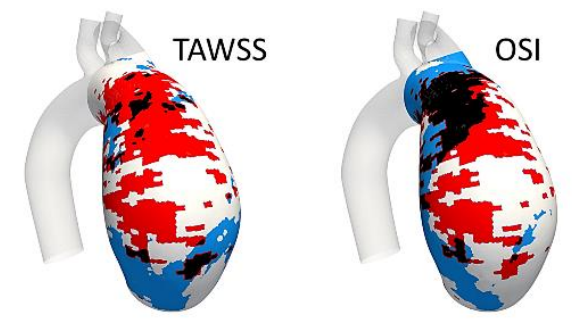

high stiffness values $\cap$ disturbed shear

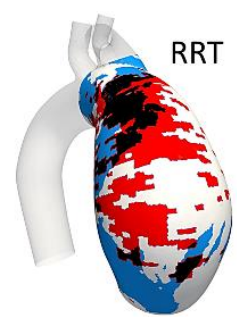

high stiffness values
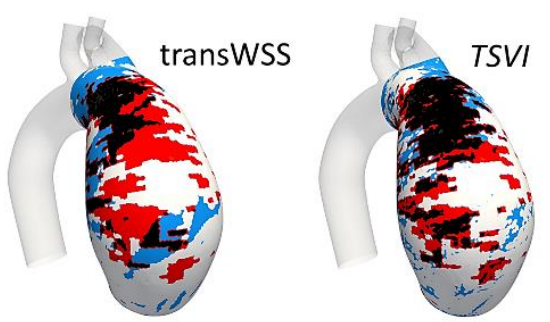

disturbed shear

(b)

755

756

\section{0}

Figure 8. Wall stiffness vs. deranged aortic hemodynamics analysis. (a) co-localization maps of high stiffness and disturbed shear; (b) similarity indices quantifying the co-localization of luminal SAs characterized by high stiffness and each descriptor of deranged hemodynamics (i.e., low TAWSS; high OSI, RRT, transWSS, TSVI).

Stiffness

OSI RRT transWSS TSVI

\section{SI}

\section{5}

